\title{
The effects of voluntary exercise on oocyte quality in a diet-induced obese murine model
}

\author{
Anna L Boudoures, Maggie Chi, Alysha Thompson, Wendy Zhang and Kelle H Moley \\ Division of Basic Science Research, Department of Obstetrics and Gynecology, Washington University in \\ St. Louis School of Medicine, 425 South Euclid Avenue, Campus Box 8064, St Louis, Missouri 63110, USA \\ Correspondence should be addressed to K H Moley; Email: moleyk@wustl.edu
}

\begin{abstract}
Obesity negatively affects many aspects of the human body including reproductive function. In females, the root of the decline in fertility is linked to problems in the oocyte. Problems seen in oocytes that positively correlate with increasing BMI include changes to the metabolism, lipid accumulation, meiosis, and metaphase II (MII) spindle structure. Studies in mice indicate that dietary interventions fail to reverse these problems. How exercise affects the oocytes has not been addressed. Therefore, we hypothesized an exercise intervention would improve oocyte quality. Here we show that in a mouse model of an exercise, intervention can improve lipid metabolism in germinal vesicle (GV) stage oocytes. Oocytes significantly increased activity and transcription of the $\beta$-oxidation enzyme hydroxyacyl-coenzyme A dehydrogenase in response to exercise training only if the mice had been fed a high-fat diet (HFD). An exercise intervention also reversed the lipid accumulation seen in GV stage oocytes of HFD females. However, delays in meiosis and disorganized MII spindles remained present. Therefore, exercise is able to improve, but not reverse, damage imparted on oocytes as a result of an HFD and obesity. By utilizing an exercise intervention on an HFD, we determined only lipid content, and lipid metabolism is changed in GV oocytes. Moving forward, interventions to improve oocyte quality may need to be more targeted to the oocyte specifically. Because of the HFD-induced deficiency in $\boldsymbol{\beta}$-oxidation, dietary supplementation with substrates to improve lipid utilization may be more beneficial. Reproduction (2016) 151 261-270
\end{abstract}

\section{Introduction}

Approximately $25 \%$ of individuals in the western world are obese, and the worldwide prevalence of obesity is predicted to continue increasing (Kelly et al. 2008). Obesity predisposes individuals to many diseases including type 2 diabetes, cardiovascular disease, and stroke (Swift et al. 2014). In addition to impairing overall health, obesity has been linked to subfertility (Bellver et al. 2010). This subfertility can likely be traced to the oocyte, as studies of oocytes obtained from women undergoing IVF demonstrated that oocytes from obese women are often apoptotic and meiotically delayed (Wittemer et al. 2000, Metwally et al. 2007).

To better understand the mechanisms causing decreased oocyte quality, many labs have modeled diet-induced obesity in mice by feeding a high-fat diet (HFD). These studies have shown that HFD affects oocyte meiotic maturation, ovulation, and fertilization and leads to embryos with restricted growth and brain abnormalities (Minge et al. 2008, Jungheim et al. 2010, Luzzo et al. 2012). Additionally, when blastocysts or two-cell embryos fertilized in HFD mice were transferred to control recipients, the resulting fetuses still had restricted growth and brain abnormalities, confirming that the defects arose from the oocytes and not the uterine environment (Sasson et al. 2014). Several papers have detailed the negative effects of HFD on oocyte quality in mice. After exposure to an HFD for only 4 weeks, oocytes of HFD mice show increased lipid accumulation, lipotoxicity, and endoplasmic reticulum stress (Wu et al. 2010). Additionally, compared to controls, HFD mice ovulate a significantly higher proportion of oocytes with disorganized meiosis II spindles (Luzzo et al. 2012). Finally, HFD oocytes have a significant increase in mitochondrial damage, including the appearance of large vacuoles and ruptured membranes, and a significant decrease in citrate production, suggesting defects in citric acid cycle metabolism (Luzzo et al. 2012).

Previously, our lab attempted to ameliorate HFDinduced oocyte damage by returning the mice to a control diet. Although overall physiology improved, including weight loss and return to normal glucose tolerance, the benefits were not paralleled in the oocytes (Reynolds et al. 2014). In humans, exercise confers many physiological benefits including decreased risk of developing cardiovascular disease, type 2 diabetes, and stroke, even in the absence of weight loss (Swift et al. 2014). Additionally, several rodent studies revealed 
that offspring of exercised dams were significantly healthier than offspring of sedentary dams (Bradley et al. 2008, Wagener et al. 2012, Carter et al. 2013, Vega et al. 2013). Finally, HFD female rats that were allowed to exercise had significant fertility improvements despite the lack of other physiological improvements (Vega et al. 2013).

Because the reproductive consequences of obesity are likely rooted at the oocyte level, and exercise is beneficial to both mother and offspring, we hypothesized that even without at change in diet, the physiological benefits of exercise would be reflected in oocytes. To test this hypothesis, we allowed HFD females to voluntarily exercise for 6 weeks and compared their oocytes to those of sedentary HFD mice and sedentary mice on a control diet. We assayed oocytes for changes in lipid accumulation, mitochondrial damage, and hydroxyacylcoenzyme A dehydrogenase (HADHA) activity (a fatty acid oxidation enzyme) and recorded the percentage of oocytes ovulated either prior to meiosis II arrest or with deformed spindles. Our data revealed that exercise was able to improve, but not entirely reverse, the negative effects of HFD on oocytes.

\section{Materials and methods}

\section{Animals and diet}

This study was carried out in strict accordance with the recommendations in the Guide for the Care and Use of Laboratory Animals of the National Institutes of Health. The protocol was approved by the IACUC-accredited Animal Studies Committee of Washington University School of Medicine (study \#20120051). Female C57BI/6J mice (Jackson Laboratories, Bar Harbor, ME, USA) were fed either an HFD (Test Diet 58R3, 58\% fat energy) or a standard mouse chow diet (CD) (Lab Diet 5053, 13\% fat energy) ad libitum from 4 weeks of age.

\section{Exercise exposure}

For the first 6 weeks of the study (age 4-10 weeks), mice were housed four per cage (feeding period). Mice were then moved to standard rat cages and housed eight mice per cage either with or without ad libitum exposure to exercise wheels (exercise exposure period) for 6 weeks. Mice remained on their respective HFD or CD during this period.

\section{Body composition analysis}

No more than $12 \mathrm{~h}$ before sacrifice, eight mice from each experimental group were subjected to body composition analysis to determine absolute fat and lean tissue mass by magnetic resonance imaging (EchoMRI 3-in-1; Echo Medical Systems, Houston, TX, USA). Two or three measurements were taken per mouse to ensure instrument precision, and values for individual mice were averaged for statistical analyses.

\section{Glucose tolerance test}

Eight to nine mice per experimental group were fasted for $12 \mathrm{~h}$ and then administered an i.p. injection of glucose ( $2 \mathrm{mg} / \mathrm{g}$ body weight) with a $30 \times 1 / 2$ gauge needle. Blood glucose was monitored by readings of a small $(2-3 \mu \mathrm{l})$ drop of tail vein blood with a one-touch Ultra Glucose Monitoring glucometer and test strips (Johnson and Johnson, New Brunswick, NJ, USA) at $15,30,60,90$, and $120 \mathrm{~min}$ after injection. Mice were then returned to their respective diets.

\section{Serum triglyceride, glucose, and insulin levels}

Eight mice in each experimental group were fasted for $4 \mathrm{~h}$ before blood was collected by puncturing the facial vein with a $25 \times 5 / 8$ gauge needle. Approximately $50 \mu \mathrm{l}$ blood was collected in BD Microtainer Serum Separator Tubes (Franklin Lakes, NJ, USA). At the same time as facial vein bleed, fasting blood glucose was measured by readings of a small $(2-3 \mu \mathrm{l})$ drop of tail vein blood with a one-touch Ultra Glucose Monitoring glucometer and test strips (Johnson and Johnson). After blood collection, mice were returned to their respective diets. Tubes were incubated for $30 \mathrm{~min}$ at room temperature (RT) and spun at $11753 \mathrm{~g}$ for $10 \mathrm{~min}$ at RT. Serum was collected on ice and stored at $-20{ }^{\circ} \mathrm{C}$ until analysis. The Infinity Triglycerides Liquid Stable Reagent Kit (Thermo Scientific, Waltham, MA, USA) was used according to the manufacturer's protocol to measure triglycerides. The Ultra Sensitive Mouse Insulin ELISA Kit (Crystal Chem, Downers Grove, IL, USA) was used according to the manufacturer's protocol to measure serum insulin levels. Each serum sample was tested in duplicate for precision, and values were averaged prior to statistical analysis.

The homeostatic model assessment of insulin resistance (HOMA1-IR) is a validated measure of IR that is calculated using the values listed in Table 1 using the following formula:

HOMA1 $-\mathrm{IR}=\frac{(\mathrm{FSI} \times \mathrm{FSG})}{22.5}$,

where fasting serum insulin (FSI, $\mathrm{mU} / \mathrm{l})$ and fasting serum glucose (FSG, mmol/l) (Matthews et al. 1985).

\section{4,4-Difluoro-4-bora-3a,4a-diaza-s-indacene 493/503 staining of germinal vesicle stage oocytes}

Three mice from each experimental condition were administered an i.p. injection of $10 \mathrm{IU}$ pregnant mare serum gonadotropin (PMSG) 46-48 h prior to sacrifice. Ovaries were removed and placed in $\mathrm{M} 2$ media $+4 \mathrm{mM}$ hypoxanthine (Sigma-Aldrich). Large antral follicles were punctured with a $29 \times 1 / 2$ gauge needle, and cumulus cells were removed by multiple passes through a glass pipette. Oocytes were then fixed in $4 \%$ paraformaldehyde (Sigma-Aldrich) diluted in PBS + 1\% polyvinylpyrrolidone (PVP; Sigma-Aldrich), washed twice, and transferred to a $1 \mu \mathrm{g} / \mathrm{ml}$ solution of 4,4-difluoro4-bora-3a,4a-diaza-s-indacene 493/503 (BODIPY; Invitrogen)+ $1 \%$ PVP for $1 \mathrm{~h}$ at RT in the dark. After two washes in PBS $+1 \%$ PVP, oocytes were mounted in VectaShield (Vector Labs, Burlingame, CA, USA) and visualized by using an Olympus 
Table 1 Serum insulin and blood glucose levels for 12-week old mice.

\begin{tabular}{|c|c|c|c|c|}
\hline & $\begin{array}{c}C D \\
\text { exercise }\end{array}$ & $\begin{array}{c}\text { CD } \\
\text { sedentary }\end{array}$ & $\begin{array}{c}\text { HFD } \\
\text { exercise }\end{array}$ & $\begin{array}{c}\text { HFD } \\
\text { sedentary }\end{array}$ \\
\hline \multicolumn{5}{|c|}{ Serum insulin $(\mathrm{ng} / \mathrm{ml})$} \\
\hline Mouse 1 & 0.48 & 0.33 & 0.74 & 0.72 \\
\hline Mouse 2 & 0.46 & 0.42 & 1.06 & 1.73 \\
\hline Mouse 3 & 0.76 & & 1.53 & 0.28 \\
\hline Mouse 4 & 0.53 & 0.55 & 1.07 & 0.87 \\
\hline Mouse 5 & 0.53 & 0.41 & 2.05 & 0.91 \\
\hline Mouse 6 & 0.50 & & 1.05 & 1.52 \\
\hline Mouse 7 & 0.50 & 0.41 & 0.69 & 1.17 \\
\hline Mouse 8 & 0.78 & 0.33 & 0.62 & 1.06 \\
\hline \multicolumn{5}{|c|}{ Blood glucose $(\mathrm{mmol} / \mathrm{l})$} \\
\hline Mouse 1 & 9.55 & 5.83 & 7.22 & 7.10 \\
\hline Mouse 2 & 6.05 & 5.83 & 6.16 & 8.49 \\
\hline Mouse 3 & 5.83 & 5.94 & 5.99 & 8.44 \\
\hline Mouse 4 & 6.27 & 4.72 & 8.49 & 8.55 \\
\hline Mouse 5 & 5.38 & 5.72 & 8.88 & 7.83 \\
\hline Mouse 6 & 5.99 & 5.88 & 6.66 & 8.66 \\
\hline Mouse 7 & 6.11 & 4.33 & 9.55 & 7.44 \\
\hline Mouse 8 & 6.66 & 5.11 & 8.44 & 9.82 \\
\hline
\end{tabular}

laser-scanning confocal microscope at $20 \times$ magnification. All images were taken as a single optical section through the plane containing the germinal vesicle (GV) at the same laser power and gain settings and adjusted by using the same gain and contrast adjustments in Adobe Photoshop CS6. For analysis, the mean gray value of pixels in an individual oocyte was measured in a minimum of 25 oocytes/group.

\section{Transmission electron microscopy}

Denuded GV stage oocytes from three mice per experimental group were collected as described for BODIPY staining and fixed in $2 \%$ paraformaldehyde $+2.5 \%$ glutaraldehyde (Polysciences, Warrington, PA, USA) in $100 \mathrm{mM}$ cacodylate buffer (Sigma-Aldrich), $\mathrm{pH} 7-7.2$, for $1 \mathrm{~h}$ at RT. Oocytes were embedded in $2 \%$ agarose and washed $3 \times 30 \mathrm{~min}$ in $100 \mathrm{mM}$ cacodylate buffer at RT. Agarose-embedded oocytes were then fixed in $1 \% \mathrm{OsO}_{4}$ (Electron Microscopy Sciences, Hartfield, PA, USA) in $100 \mathrm{mM}$ cacodylate buffer for $1 \mathrm{~h}$ at RT. Oocytes were washed thoroughly in distilled water, stained in $1 \%$ uranyl acetate in $\mathrm{dH}_{2} \mathrm{O}$ for $1 \mathrm{~h}$ at $\mathrm{RT}$, dehydrated in a series of ethanol washes, and then washed in $100 \%$ ethanol at RT. Next, oocytes were washed twice in propylene oxide (Electron Microscopy Sciences) and then infiltrated with resin by using the Eponate 12 Kit (Ted Pella, Redding, CA, USA). After infiltration, blocks were embedded, cured, and then sectioned with a Leica Ultracut UCT Ultramicrotome (Leica Microsystems, Inc., Bannockburn, IL, USA). Sections $(90 \mathrm{~nm})$ were stained with uranyl acetate and Reynolds' lead citrate before viewing at $2000 \times-15000 \times$ magnification with a JEOL 1200 EX Transmission Electron Microscope (JEOL USA, Inc., Peabody, MA, USA) equipped with an AMT eight megapixel digital camera (Advanced Microscopy Techniques, Woburn, MA, USA). Images taken at $15000 \times$ magnification were blinded and analyzed by three independent observers for mitochondrial shape, the number of lipid droplets in each image, and the number of rose petal mitochondria in each image.

\section{HADHA type 2 enzymatic activity}

Denuded GV stage oocytes were collected from three mice per group as described for BODIPY staining. Individual oocytes were frozen on glass slides in isopentane equilibrated with liquid nitrogen and freeze-dried overnight under vacuum at $-35{ }^{\circ} \mathrm{C}$. In total, 15 individual oocytes were extracted under nanoliter volume and used for analysis of levels of HADHA activity by enzyme-linked cycling assays as previously described (Chi et al. 2002).

\section{Quantitative real-time PCR}

Three GV oocytes per mouse were collected from six mice per experimental group as described for BODIPY staining. Oocytes were denuded and washed in $50 \mathrm{mg} / \mathrm{ml}$ nuclease-free BSA (Invitrogen) on ice. Oocytes were then transferred to the single cell lysis solution with single cell DNase I solution (both parts of the Single Cell-To-Ct Kit (Ambion, Austin, TX, USA)). All steps of RNA isolation and RT followed the manufacturer's protocol. Real-time PCRs were carried out on a 7500 fast real-time PCR system. Expression of carnitine palmitoyltransferase 2 (Cpt2), Hadh, aconitase 2 (Aco2), isocitrate dehydrogenase $3 \mathrm{~b}(I d h 3 b)$, and $A c t b$ were measured by using the inventoried TaqMan Gene Expression Assays (Cpt2: Mm00487205_m1; Hadh: Mm00492535_m1; and Actb: Mm00607939_s1; Life Technologies). Expression of genes for each diet was normalized to Actb based on its utilization in prior publications as a reference gene in oocytes, and its expression remains stable throughout meiosis (Bachvarova et al. 1985).

\section{Spindle and chromosome imaging of metaphase II stage oocytes}

Four to five mice from each of the four experimental conditions were i.p. injected with $10 \mathrm{IU}$ of PMSG (Sigma-Aldrich) and then with $5 \mathrm{IU}$ of human chorionic gonadotropin (hCG; Sigma-Aldrich) $48 \mathrm{~h}$ after PMSG. Mice were sacrificed by cervical dislocation 12-14 h after hCG administration, and the cumulus-oocyte complexes (COCs) were collected from the ampullae. Cumulus cells were removed with a brief wash in $1 \mathrm{mg} / \mathrm{ml}$ hyaluronidase in M2 media (Sigma-Aldrich). Oocytes were then fixed for $30 \mathrm{~min}$ in $4 \%$ paraformaldehyde in PBS $+1 \%$ BSA (Sigma-Aldrich). Oocytes were transferred to a permeabilization solution $(0.5 \%$ Triton $\mathrm{X}-100$ in PBS) for 20 min and then blocked with PBS $+0.1 \%$ Tween- $20+0.01 \%$ Triton $\mathrm{X}-100+1 \% \mathrm{BSA}$ for $1 \mathrm{~h}$. Oocytes were stained with FITC-conjugated anti-tubulin antibody (1:500; Sigma-Aldrich) overnight at $4{ }^{\circ} \mathrm{C}$, washed three times, and then co-stained with TOPRO-3 iodide (1:500; Invitrogen) for $10 \mathrm{~min}$. Oocytes were washed twice in PBS $+0.1 \%$ Tween-20+0.01\% Triton X-100, mounted in VectaShield, and visualized by using an Olympus laser-scanning confocal microscope at $20 \times$ magnification. Images were blinded and analyzed by three independent observers for meiotic stage (metaphase II (MII) vs prophase or anaphase), spindle shape (normal, barrel shaped vs deformed), and apoptosis. 


\section{Results}

\section{Exercise training improves some aspects of whole-body physiology}

Previously, our lab and others have shown that mice maintained on a HFD became obese and had damaged oocytes. To determine whether exposure to voluntary exercise could reverse any of the defects, we exposed mice to either HFD or control CD for 12 weeks beginning at 4 weeks of age. During the last 6 weeks, half of each diet cohort had access to an exercise wheel $24 \mathrm{~h}$ a day, based on the experimental design of Schulkey et al. (exercise exposure period). Mice were filmed for a 24-h period to confirm frequent use of wheels (data not shown). We found that HFD exposure caused significant weight gain that was not reversible by exercise $(n=32$ /group, Fig. 1A). However, compared to their sedentary counterparts of the same age, the cohort of HFD exercising mice had significantly less fat mass and unchanged lean mass ( $n=8$ /group, Fig. $1 \mathrm{~B}$ and C). This significant change in fat mass was likely not reflected in total body mass because of the differences in sample size. HFD mice had significantly higher IR as measured
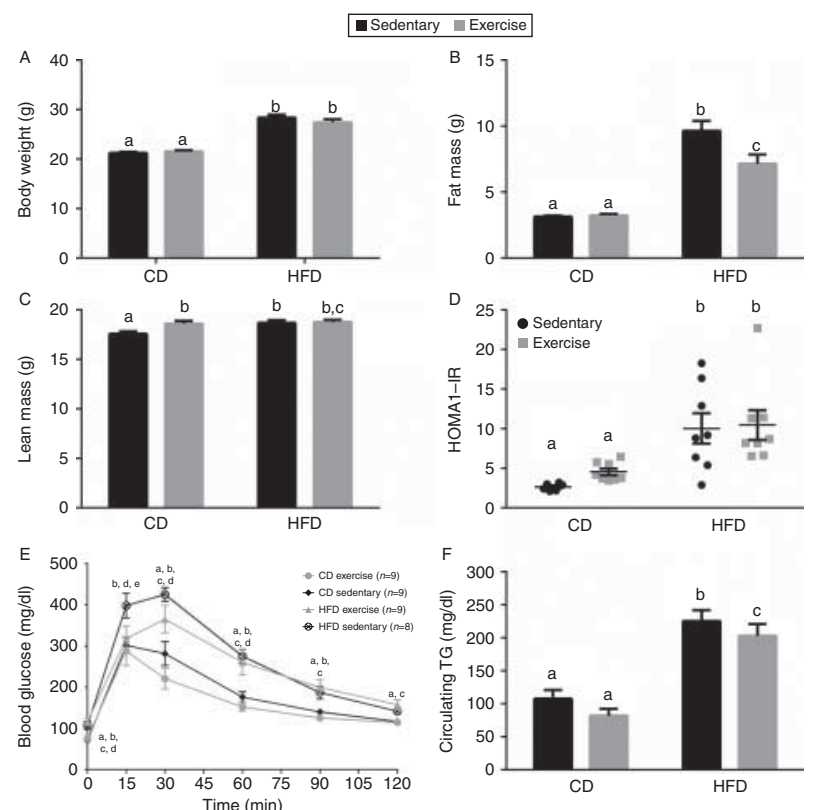

Figure 1 Body weights and body composition and metabolic parameters of mice. (A) Average body weights, (B) fat mass, and (C) lean mass as measured by magnetic resonance imaging. (D) homeostatic model assessment of insulin resistance (HOMA1-IR) calculated as described in 'Materials and methods' section. (E) Glucose tolerance test, (F) fasting serum triglycerides. (A, B, C, D, E and F) Different letters designate statistical significance between groups whereas same letter indicates no significance between groups, $P<0.05$. (E) Letters above each time point represent statistical significance between groups at that time as follows: $a, C D$ exercise vs HFD exercise; $b, C D$ exercise vs HFD sedentary; c, CD sedentary vs HFD exercise; $d$, CD sedentary vs HFD sedentary; and e, HFD exercise vs HFD sedentary, $P<0.05$. All data was analyzed by two-way ANOVA with Tukey-Kramer correction for multiple comparisons. CD, chow diet; HFD, high-fat diet. by the HOMA-IR, which was not reversed by exercise (Fig. 1D). Additionally, the range of insulin sensitivity was much greater in HFD mice than CD mice. Allowing mice to exercise while maintaining a HFD was able to cause a trend toward a restoration of glucose tolerance, especially at earlier time points (see 15-min time point in Fig. 1E). Exercise was able to significantly decrease triglyceride levels in HFD females, but triglyceride levels were still higher than in CD mice; exercise did not significantly change triglyceride levels in $C D$ mice (Fig. 1F). Therefore, our results confirm previously published findings that exercise alone cannot improve body weight, but some physiological parameters are improved by exercise (Kelly et al. 2011, Carter et al. 2013, Vega et al. 2013, Stanford et al. 2014).

\section{Changes in oocyte lipid accumulation}

Human obesity causes an increased accumulation of triglycerides in the follicular fluid surrounding the oocyte in a maturing ovarian follicle, and mouse oocytes exposed to this lipid-rich environment also contain excess lipids (Jungheim et al. 2011, Yang et al. 2012, Dunning et al. 2014a,b, Valckx et al. 2014a,b). Thus, given that exercising HFD mice had lower serum triglycerides than their sedentary peers, we hypothesized that these mice would also have decreased lipid accumulation in their maturing oocytes. To test this, oocytes were collected from superovulated females at the GV stage, stained with the fluorescent neutral lipid dye BODIPY 493/503, and imaged by confocal microscopy (Fig. 2A). Measurements of the mean gray value of the fluorescence of individual oocytes revealed that, as expected, oocytes from sedentary HFD females contained more lipid than oocytes from CD females. However, oocytes from exercised HFD females contained the same amount of lipid as those from sedentary $C D$ females. Surprisingly, oocytes from exercised $C D$ mice contained the most lipid of any of the groups (Fig. 2A and B).

We also examined lipid accumulation by counting lipid droplets in transmission electron microscopy (TEM) images of GV oocytes. Consistent with the BODIPY results, oocytes from sedentary HFD females contained more lipid droplets than those from exercised HFD mice or either of the CD groups (Fig. 2C and D). From these data, we conclude that exercise is able to reverse the lipid accumulation phenotype induced by exposure to HFD.

\section{Oocyte metabolism is affected by changes in diet and exercise}

Oocytes rely heavily on carbohydrates supplied by cumulus cells to generate ATP and fuel maturation and fertilization (Rieger \& Loskutoff 1994). In addition, 
A
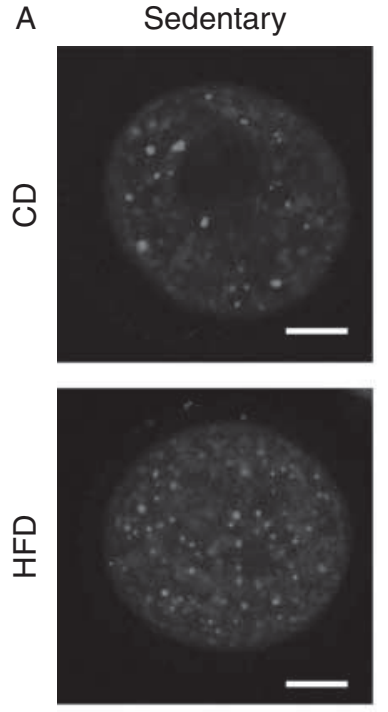

B

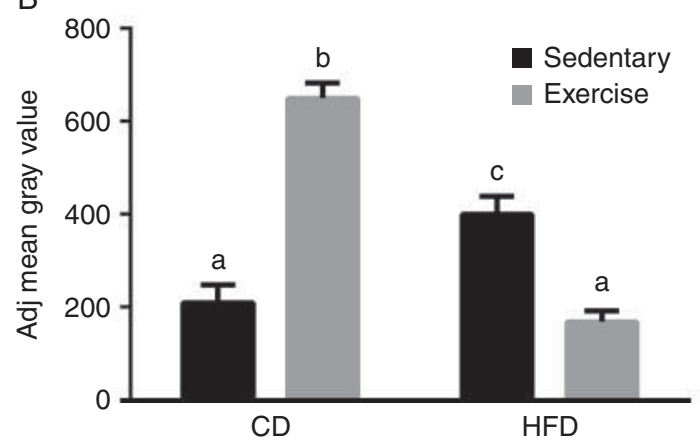

C
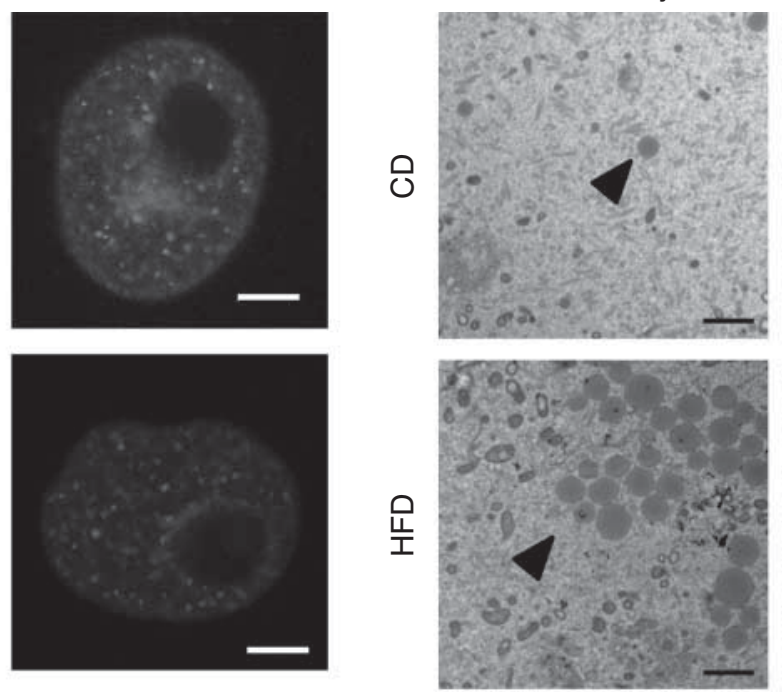

D

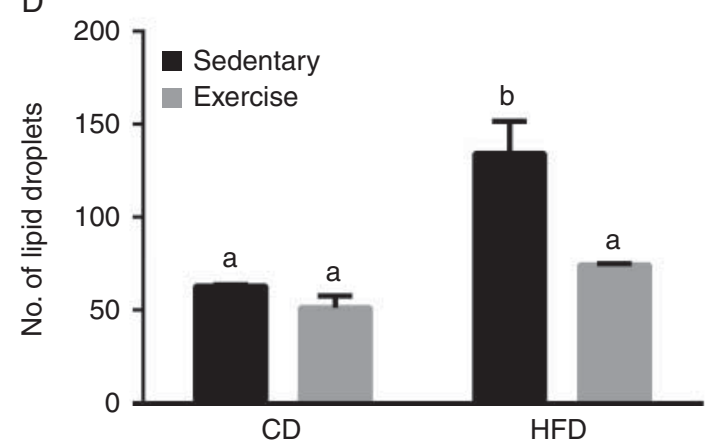

Figure 2 Lipid droplet accumulation in germinal vesicle stage oocytes in response to diet and exercise training. (A) Representative images of oocytes from BODIPY 493/503 staining. All images were taken at the same keeping confocal settings identical, scale bars: $25 \mu \mathrm{m}$. (B) Mean relative gray value of fluorescence of individual oocyte area measured using ImageJ (Rasabanad, WS). (C) Representative images of sections of oocytes taken by transmission electron microscopy at $2000 \times$ magnification, scale bars: $2 \mu \mathrm{m}$. Black arrowheads indicate lipid droplets. (D) Total number of lipid droplets counted in images from each group. Different letters designate statistical significance and same letter indicates no significance between groups, $P<0.05$; two-way ANOVA with Tukey-Kramer correction for multiple comparisons.

maturing oocytes metabolize lipids by the fatty acid oxidation pathway within the mitochondria (Dunning et al. 2010, 2014b). Thus, the significant increase of lipids in the GV oocytes of sedentary HFD females led us to ask whether these oocytes have changes in their mitochondrial metabolism. First, the significant changes in lipid accumulation after exercise exposure led us to hypothesize that there may be changes to fatty acid metabolism. To test this, we used enzymatic cycling to measure activity of HADHA in individual GV stage oocytes. HADHA is a key enzymatic subunit of the mitochondrial trifunctional protein, which catalyzes three steps of fatty acid $\beta$-oxidation (Houten \& Wanders 2010). We found that oocytes from both cohorts of HFD females had significantly lower HADHA activity than oocytes from both cohorts of $\mathrm{CD}$ females. Although exercise was not able to increase the level of HADHA activity in HFD oocytes to the levels observed in CD oocytes, oocytes from exercised HFD and CD mice had significantly higher HADHA activity than those of their sedentary counterparts (Fig. 3A).

To determine if this activity change was reflected in ATP production, we next measured ATP content of individual GV oocytes using enzymatic cycling assays. However, we observed significantly lower ATP only in HFD exercising mice (Fig. 3B). Lower ATP levels contradict the positive effects of exercise on HADHA activity. Fatty acid oxidation produces 14 molecules of ATP for every two carbons in a carbon chain (e.g. 104 molecules of ATP for a single molecule of palmitate). Given the efficiency of this reaction, increased HADHA activity would be expected to increase ATP (Garrett \& Grisham 2013). To decide if the contradiction of HADHA activity and ATP production could be explained by changes to other metabolic pathways, we measured citrate levels in oocytes (Fig. 3C). Citrate 

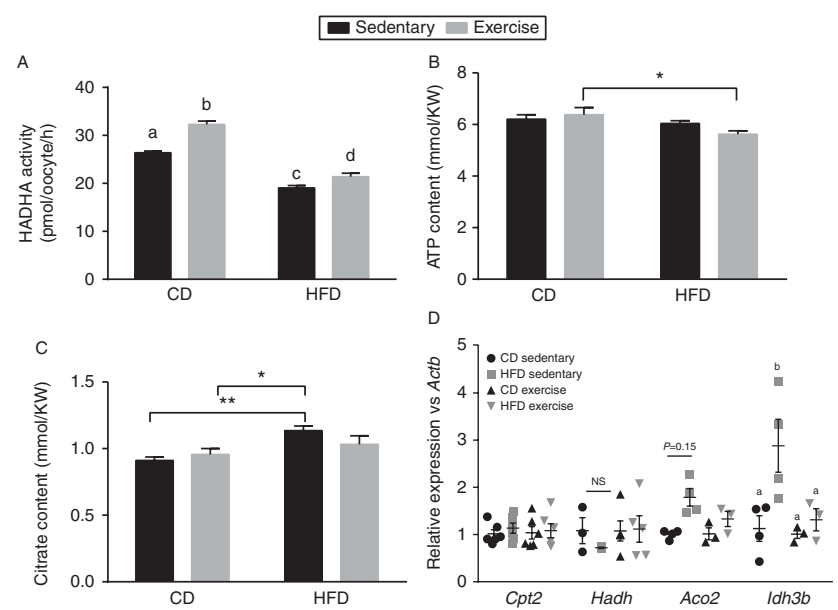

Figure 3 Metabolic enzyme activity, metabolite levels, and transcript levels are altered by diet and exercise. (A) HADHA enzymatic activity was measured in individual GV stage oocytes ( $n=15$ oocytes/group). Two-way ANOVA with multiple comparisons, different letters represent statistical significance between groups, $P<0.001$. (B) ATP and (C) citrate content of GV stage oocytes as measured by enzymatic cycling assay ( $n=45$ oocytes/group). Two-way ANOVA with multiple comparisons; ${ }^{*} P<0.05$ and ${ }^{* *} P<0.01$. (D) Relative abundance of Cpt2, Hadh, Idh3b, and Aco2 transcripts; three oocytes per mouse were collected from six individual mice per experimental condition, and relative abundance of transcripts was calculated using the $\Delta \Delta C_{\mathrm{T}}$ method, with Actb as the reference gene. Two-way ANOVA with multiple comparisons; for Idh $3 b$ : a, no change between groups and b, $P<0.001$ for Hadh sedentary vs exercise. For Aco2, the difference was not statistically significant but is trending toward significance $(P=0.015)$.

was significantly higher only in oocytes from HFD sedentary females. These results are both surprising and conflicting to both the activity of HADHA and ATP levels of oocytes.

Although the data are conflicting, these data agree with transcript abundance of TCA cycle enzymes and HADHA; HFD sedentary mice have significantly higher levels Idh $3 \mathrm{~b}$, and levels of Aco2 are increased, but the results did not reach statistical significance $(P=0.15)$, two enzymes involved in the TCA cycle (Fig. 3D). This is likely due to increased citrate abundance. Similarly, the transcript abundance of fatty acid oxidation enzyme HADHA is significantly decreased only in HFD sedentary oocytes. This is reflected in the significant decrease in HADHA activity and lipid droplet accumulation also observed in HFD sedentary oocytes (Figs 2 and 3A respectively).

\section{Mitochondrial ultrastructure is changed by exercise exposure}

Because metabolite and qPCR data indicated changes to mitochondrial pathways, but could not fully explain the changes to lipid accumulation observed in oocytes form HFD sedentary and exercise mice, we wondered whether mitochondrial damage previously reported persisted or was changed by exercise (Wu et al. 2010, Luzzo et al. 2012). To address this question, we used TEM to examine mitochondrial ultrastructure in GV stage oocytes. Whereas oocyte mitochondria are normally round, the appearance of elliptical and dumbbell-shaped mitochondria suggests the occurrence of fission and fusion (Friedman \& Nunnari 2014). We found that oocytes from sedentary HFD females had a higher proportion of elliptical and dumbbell-shaped mitochondria than did oocytes from exercising HFD mice or CD mice (Fig. 4A and B).

Another morphological measure of mitochondrial health is the appearance of so-called 'rose petal' mitochondria (see examples in Fig. 4C). These occur when there are deficiencies in oligomerization of the $\mathrm{F}_{1} \mathrm{~F}_{0}$-ATP synthase in the mitochondrial inner membrane (Arselin et al. 2003). To assess presence of rose petal mitochondria in the four cohorts of mice, we collected GV oocytes from three mice from each group and subjected them to TEM. Images were coded and scored by three independent blinded observers. We found that oocytes from sedentary HFD mice had significantly more rose petal mitochondria than did oocytes from $C D$ mice. However, oocytes from exercised HFD mice had similar numbers of rose petal mitochondria as CD mice (Fig. 4D). These results suggest that oocyte mitochondrial damage that occurs as a result of HFD exposure can be compensate for by exercise.

\section{MII spindle structure is unchanged by exercise}

Exposure to a HFD causes multiple abnormalities in oocytes including disorganized spindle structure in the MII oocyte, aberrant segregation of chromosomes, and delayed meiotic maturation (Luzzo et al. 2012). Lipid accumulation was also linked to delayed oocyte maturation and increased apoptosis at the MII stage (Wu et al. 2010). Consistent with these reports, we observed a greater proportion of delayed oocytes in HFD mice than in CD mice $(C D$ sedentary $=15 \%$; $C D$ exercise $=7 \%$ vs HFD sedentary $=38 \%$; and HFD exercise $=32 \%$; Fig. 5). However, the proportion of normal, meiotically mature oocytes from exercised HFD mice did not differ greatly from those of sedentary HFD mice (HFD exercise $=40 \%$ and HFD sedentary $=32 \%$; Fig. 5).

\section{Discussion}

Here, we report that exercise brought about moderate change to female physiology and a significant decrease in lipid accumulation in the oocytes of HFD females. This may be due, in part, to increased activity of the fatty acid oxidation enzyme HADHA. However, these positive changes did not negate the effects of HFD on spindle morphology and meiotic maturation at ovulation. 
A

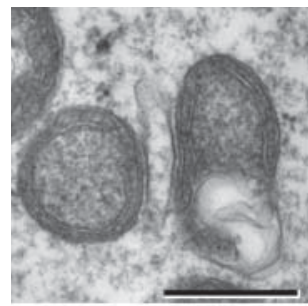

Round and elliptical shape

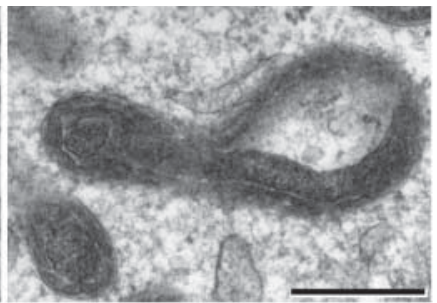

Dumbbell shape

B

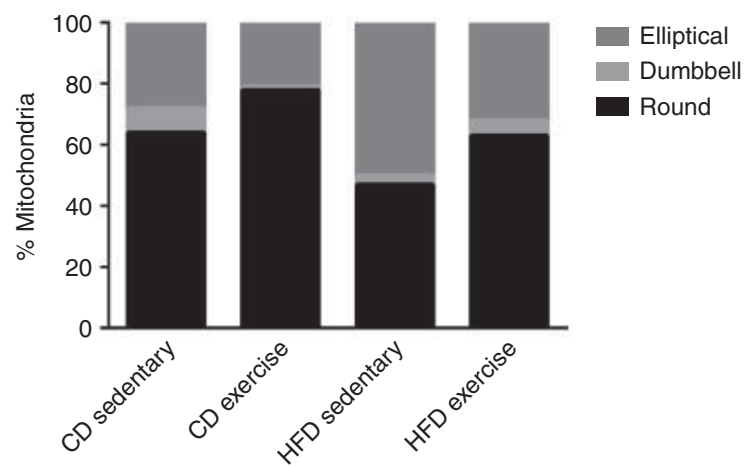

C

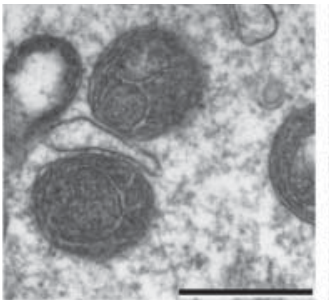

Normal mitochondria

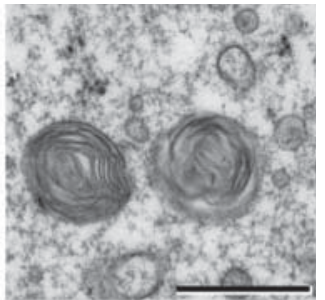

Rose petal mitochondria

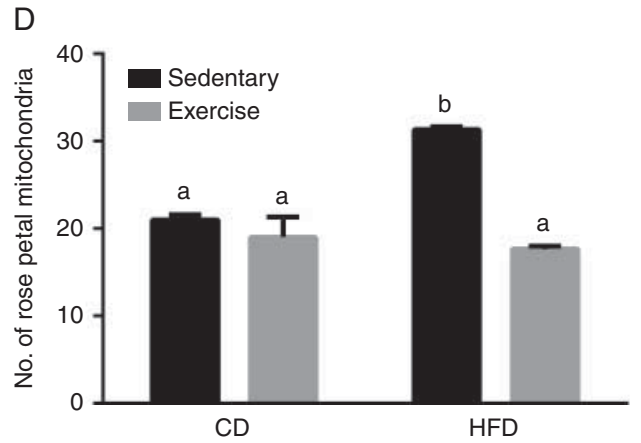

Figure 4 Transmission electron microscopy of GV stage oocytes. (A) Representative image of round (normal), elliptical, and dumbbell-shaped oocyte mitochondria. (B) Percentages of shape of mitochondria from each cohort of mice ( $n=7-9$ oocytes/cohort). (C) Representative image of normal oocyte mitochondria and rose petal mitochondria. (D) Comparison of the number of rose petal mitochondria counted in oocytes from each cohort ( $n=7-9$ oocytes/cohort), two-way ANOVA with multiple comparisons, different letters designate statistical significance and same letter indicates no significance between groups, $P<0.05$. All images are at $15000 \times$ magnification, scale bars: $500 \mathrm{~nm}$.

We found that, in female mice, voluntary exercise resulted in moderate physiological benefits and no significant total body weight loss. This is not surprising for three reasons. First, in a study of mice that were forced to exercise, female mice had a much smaller decrease in total body mass and fat mass than their male counterparts (Kelly et al. 2010). Secondly, the mice in our study voluntarily exercised, likely at lower levels than those in the forced exercise study. Although we did not control for the exact amounts of exercise each female received, our experimental design was based off of a study that showed moderate voluntary exercise in HFD females which was beneficial in the reversal of congenital heart disease in their offspring (Schulkey et al. 2015). Finally, a weak relationship between exercise and weight loss has also been reported in humans (Swift et al. 2014).

Although body weight was largely unchanged by exposure to exercise, the lipid content of oocytes from HFD females was decreased, as shown by both our BODIPY and EM data. Although we saw more lipid in the oocytes of exercising CD mice than in their sedentary counterparts, our contrasting EM data suggest that this was due to experimental error. Oocytes were used from three mice and pooled prior to staining, thus contribution from any one mouse is not possible to determine.
Our data are consistent with previous findings that lipid accumulates in oocytes in the presence of increased substrate availability both in vivo (Wu et al. 2010, Luzzo et al. 2012) and in vitro (Jungheim et al. 2011), but the mechanism of lipid uptake by oocytes is still under investigation.

The exercise-induced reductions in lipid content of oocytes we observed in HFD mice might, in part, be explained by the significant decrease in circulating serum triglycerides in the mice. Previous research in both mice and humans has shown that serum levels of triglycerides correlate with triglyceride levels in both the ovarian follicle and the oocyte (Wu et al. 2012, Yang et al. 2012).

The accumulation of lipids within oocytes of HFD females and the reductions we recorded after exercise exposure may also be partially explained by changes to expression of TCA cycle enzymes and fatty acid $\beta$-oxidation. We found that the transcript abundance and activity level of the fatty acid $\beta$-oxidation enzyme HADHA was lower in oocytes of sedentary HFD mice than in all other groups of mice. Additionally, HFD sedentary mice had at significant increase in TCA cycle enzyme Idh3, and transcript levels of Aco2 were increased, but the difference was not statistically significant. However, exercise exposure significantly 


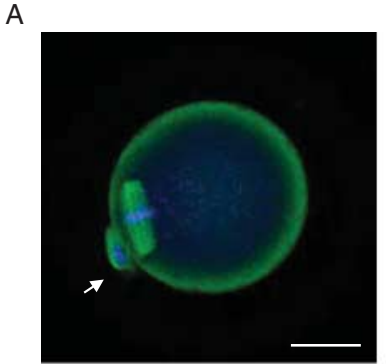

Normal

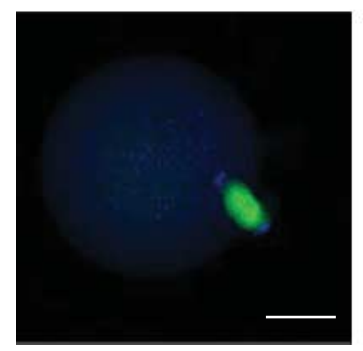

Delayed

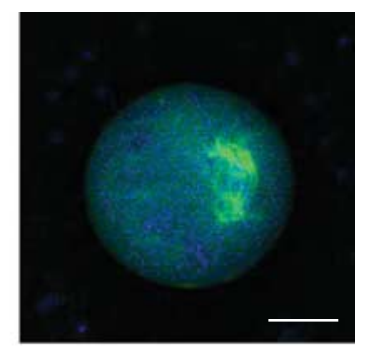

Abnormal

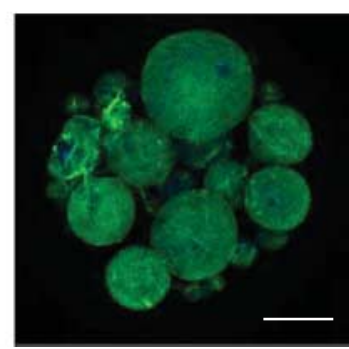

Apoptotic

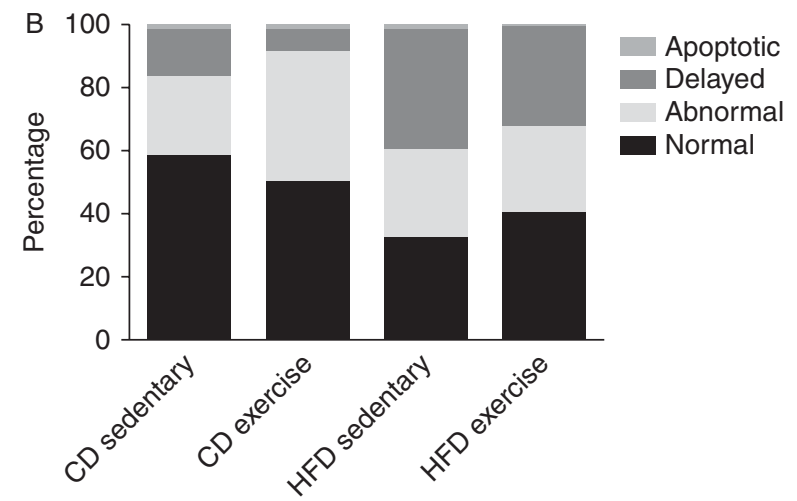

Figure 5 Meiotic progression and spindle structure of metaphase II (MII) stage oocytes. (A) Representative images of each classification category of meiotic anomalies. Arrow indicates polar body. Apoptotic indicates and apoptotic oocyte contained within the zona pellucida, scale bars: $25 \mu \mathrm{m}$. (B) Graph depicting percentage of oocyte in each category from each of the four experimental conditions.

increased HADHA activity and decreased Idh3 gene expression changes in oocytes from both the HFD mice. One explanation for the increase in TCA cycle gene expression and decrease fatty acid oxidation gene expression genes is that there is chronic utilization of the TCA cycle due to the high glucose levels in the blood. In a study of patients with non-alcoholic fatty liver disease, investigators showed that there is a chronic utilization of the TCA cycle due to increased blood glucose, which results in no utilization of lipid and causes the triglycerides to accumulate in hepatocytes (Sunny et al. 2011). Our qPCR, metabolite and HADHA activity assays, in combination with the rescue of the HFD sedentary increase lipid, suggests a similar mechanism could be occurring in the oocytes. However, further functional assays not conducted here must be conducted to confirm this.

This finding is in agreement with previous demonstrations that exercise increases fatty acid oxidation in mitochondria of other tissue types (Houten \& Wanders 2010). Additionally, mitochondrial biogenesis is stimulated in the skeletal muscle of exercised animals (Russell et al. 2014), but we have not explored that possibility here.

Fatty acid $\beta$-oxidation is necessary for proper oocyte maturation and ovulation and embryo development (Dunning et al. 2010). For example, inhibition of $\beta$-oxidation in cumulus-enclosed oocytes prevents the oocytes from eliciting the appropriate hormone-induced meiotic activation and maturation in vitro (Valsangkar \& Downs 2013). Additionally, defects in $\beta$-oxidation cause fertility defects in mice and humans (Dunning et al. 2014a). Finally, enhancing fatty acid oxidation by exposure of oocytes to L-carnitine improves the blastocyst development rate after fertilization (Paczkowski et al. 2014). Thus, an important question for the future is whether or not the increase in fatty acid oxidation activity induced by exercise can improve fertility.

The increased HADHA activity correlated with improvements to two aspects of mitochondrial ultrastructure: fewer rose petal mitochondria and fewer elliptical mitochondria. In oocytes, mitochondrial ultrastructure is generally round with cristae that wrap the periphery (Sathananthan \& Trounson 2000). In other tissue types, particularly skeletal muscle, both exercise training and IR cause changes to mitochondrial ultrastructure and activity (Yan et al. 2012, Russell et al. 2014). Here, our data indicate that exercise may be able to induce changes to mitochondrial morphology independent of IR. HOMA-IR was unaffected by moderate exercise, yet exercise reduced the prevalence of abnormal elliptical shaped mitochondria by EM analysis. A direct relationship between glucose tolerance, IR, and mitochondrial shape was not addressed here. Still, previous research suggests impaired glucose tolerance, and insulin responsiveness can affect mitochondrial morphology (Wang et al. 2009, 2012, Luzzo et al. 2012). While the mechanism underlying the changes in mitochondrial morphology remains to be determined, we demonstrate here that exercise is clearly beneficial and may be able to override the previously observed negative effects of IR on oocyte mitochondria morphology (Wang et al. 2009).

Despite the benefits of moderate exercise on lipid accumulation and mitochondrial structure in the oocytes of HFD mice, exercise did not change the proportion of oocytes ovulated with deformed spindles. One possibility is that glucose tolerance is not completely normalized by exercise, and IR is unchanged by exercise. Glucose intolerance due to IR could lead to decreased glucose uptake within the COCs during maturation. Previously, it has been shown glucose uptake by COCs is an insulin-stimulated response, 
meaning it is less sensitive in situations in cases of $I R$ and decreased glucose tolerance (Purcell et al. 2012). Additionally, IR and glucose intolerance in mice attenuates insulin-stimulated glucose uptake in oocytes (Wang et al. 2012). Importantly, the decreased glucose uptake correlated with deformed spindles. Therefore, a possible explanation for unchanged spindle morphology is persistent impaired glucose tolerance and IR in HFD mice after exercise exposure.

In conclusion, we showed that exercise was able to improve some aspects of oocyte quality in HFD-exposed mice. Whether these improvements to oocyte quality are beneficial to fertility remains to be investigated. Likewise, it will be important to determine whether exercise can improve oocyte quality in obese women who are attempting to conceive.

\section{Declaration of interest}

The authors declare that there is no conflict of interest that could be perceived as prejudicing the impartiality of the research reported.

\section{Funding}

This work was supported by a grant from the National Institutes of Health, R01HD083895, and a grant from the American Diabetes Association, research grant 7624.

\section{Acknowledgements}

The authors thank Deborah Frank, PhD, Obstetrics and Gynecology, Washington University in St Louis, for critical reading and editing of this manuscript.

\section{References}

Arselin G, Giraud MF, Dautant A, Vaillier J, Brethes D, Coulary-Salin B, Schaeffer J \& Velours J 2003 The GxxxG motif of the transmembrane domain of subunit e is involved in the dimerization/oligomerization of the yeast ATP synthase complex in the mitochondrial membrane. European Journal of Biochemistry 270 1875-1884. (doi:10.1046/ j.1432-1033.2003.03557.x)

Bachvarova R, De Leon V, Johnson A, Kaplan G \& Paynton BV 1985 Changes in total RNA, polyadenylated RNA, and actin mRNA during meiotic maturation of mouse oocytes. Developmental Biology 108 325-331. (doi:10.1016/0012-1606(85)90036-3)

Bellver J, Ayllon Y, Ferrando M, Melo M, Goyri E, Pellicer A, Remohi J \& Meseguer M 2010 Female obesity impairs in vitro fertilization outcome without affecting embryo quality. Fertility and Sterility 93 447-454. (doi:10.1016/j.fertnstert.2008.12.032)

Bradley RL, Jeon JY, Liu FF \& Maratos-Flier E 2008 Voluntary exercise improves insulin sensitivity and adipose tissue inflammation in dietinduced obese mice. American Journal of Physiology. Endocrinology and Metabolism 295 E586-E594. (doi:10.1152/ajpendo.00309.2007)

Carter LG, Qi NR, De Cabo R \& Pearson KJ 2013 Maternal exercise improves insulin sensitivity in mature rat offspring. Medicine and Science in Sports and Exercise 45 832-840. (doi:10.1249/MSS. ob013e31827de953)
Chi MM, Hoehn A \& Moley KH 2002 Metabolic changes in the glucoseinduced apoptotic blastocyst suggest alterations in mitochondrial physiology. American Journal of Physiology. Endocrinology and Metabolism 283 E226-E232. (doi:10.1152/ajpendo.00046.2002)

Dunning KR, Cashman K, Russell DL, Thompson JG, Norman RJ \& Robker RL 2010 Beta-oxidation is essential for mouse oocyte developmental competence and early embryo development. Biology of Reproduction 83 909-918. (doi:10.1095/biolreprod.110.084145)

Dunning K, Russell DL \& Robker R 2014a Lipids and oocyte developmental competence: the role of fatty acids and $\beta$-oxidation. Reproduction 148 R15-R27. (doi:10.1530/REP-13-0251)

Dunning KR, Anastasi MR, Zhang VJ, Russell DL \& Robker RL 2014b Regulation of fatty acid oxidation in mouse cumulus-oocyte complexes during maturation and modulation by PPAR agonists. PLOS ONE 9 e87327. (doi:10.1371/journal.pone.0087327)

Friedman JR \& Nunnari J 2014 Mitochondrial form and function. Nature 505 335-343. (doi:10.1038/nature12985)

Garrett R \& Grisham CM 2013 Biochemistry, 5th edn. Belmont, CA: Brooks/Cole, Cengage Learning.

Houten SM \& Wanders RJ 2010 A general introduction to the biochemistry of mitochondrial fatty acid $\beta$-oxidation. Journal of Inherited Metabolic Disease 33 469-477. (doi:10.1007/s10545-010-9061-2)

Jungheim ES, Schoeller EL, Marquard KL, Louden ED, Schaffer JE \& Moley KH 2010 Diet-induced obesity model: abnormal oocytes and persistent growth abnormalities in the offspring. Endocrinology 151 4039-4046. (doi:10.1210/en.2010-0098)

Jungheim ES, Louden ED, Chi MM, Frolova AI, Riley JK \& Moley KH 2011 Preimplantation exposure of mouse embryos to palmitic acid results in fetal growth restriction followed by catch-up growth in the offspring. Biology of Reproduction 85 678-683. (doi:10.1095/biolreprod.111. 092148)

Kelly T, Yang W, Chen CS, Reynolds K \& He J 2008 Global burden of obesity in 2005 and projections to 2030. International Journal of Obesity 32 1431-1437. (doi:10.1038/ijo.2008.102)

Kelly SA, Nehrenberg DL, Hua K, Gordon RR, Garland T Jr \& Pomp D 2010 Parent-of-origin effects on voluntary exercise levels and body composition in mice. Physiological Genomics 40 111-120. (doi:10.1152/ physiolgenomics.00139.2009)

Kelly SA, Nehrenberg DL, Hua K, Garland T Jr \& Pomp D 2011 Exercise, weight loss, and changes in body composition in mice: phenotypic relationships and genetic architecture. Physiological Genomics 43 199-212. (doi:10.1152/physiolgenomics.00217.2010)

Luzzo KM, Wang Q, Purcell SH, Chi M, Jimenez PT, Grindler N, SchedI T \& Moley KH 2012 High fat diet induced developmental defects in the mouse: oocyte meiotic aneuploidy and fetal growth retardation/brain defects. PLOS ONE 7 e49217. (doi:10.1371/journal. pone.0049217)

Matthews DR, Hosker JP, Rudenski AS, Naylor BA, Treacher DF \& Turner RC 1985 Homeostasis model assessment: insulin resistance and $\beta$-cell function from fasting plasma glucose and insulin concentrations in man. Diabetologia 28 412-419. (doi:10.1007/BF00280883)

Metwally M, Cutting R, Tipton A, Skull J, Ledger WL \& Li TC 2007 Effect of increased body mass index on oocyte and embryo quality in IVF patients. Reproductive Biomedicine Online 15 532-538. (doi:10.1016/S14726483(10)60385-9)

Minge CE, Bennett BD, Norman RJ \& Robker RL 2008 Peroxisome proliferator-activated receptor-gamma agonist rosiglitazone reverses the adverse effects of diet-induced obesity on oocyte quality. Endocrinology 149 2646-2656. (doi:10.1210/en.2007-1570)

Paczkowski M, Schoolcraft WB \& Krisher RL 2014 Fatty acid metabolism during maturation affects glucose uptake and is essential to oocyte competence. Reproduction 148 429-439. (doi:10.1530/REP14-0015)

Purcell SH, Chi MM, Lanzendorf S \& Moley KH 2012 Insulin-stimulated glucose uptake occurs in specialized cells within the cumulus oocyte complex. Endocrinology 153 2444-2454. (doi:10.1210/en. 2011-1974)

Reynolds K, Boudoures AL, Chi M, Wang Q \& Moley K 2014 The adverse effect of obesity/high fat diet on oocyte quality and metabolism is not reversible with resumption of regular diet in mice. Reproduction, Fertility, and Development 27 716-724. (doi:10.1071/RD14251) 
Rieger D \& Loskutoff NM 1994 Changes in the metabolism of glucose, pyruvate, glutamine and glycine during maturation of cattle oocytes in vitro. Journal of Reproduction and Fertility 100 257-262. (doi:10.1530/ jrf.0.1000257)

Russell AP, Foletta VC, Snow RJ \& Wadley GD 2014 Skeletal muscle mitochondria: a major player in exercise, health and disease. Biochimica et Biophysica Acta 1840 1276-1284. (doi:10.1016/j.bbagen.2013.11.016)

Sasson IE, Vitins AP, Mainigi MA, Moley KH \& Simmons RA 2014 Pre-gestational vs gestational exposure to maternal obesity differentially programs the offspring in mice. Diabetologia 58 615-624. (doi:10.1007/ s00125-014-3466-7)

Sathananthan AH \& Trounson AO 2000 Mitochondrial morphology during preimplantational human embryogenesis. Human Reproduction 15 (Suppl 2) 148-159. (doi:10.1093/humrep/15.suppl_2.148)

Schulkey CE, Regmi SD, Magnan RA, Danzo MT, Luther H, Hutchinson AK, Panzer AA, Grady MM, Wilson DB \& Jay PY 2015 The maternal-ageassociated risk of congenital heart disease is modifiable. Nature $\mathbf{5 2 0}$ 230-233. (doi:10.1038/nature14361)

Stanford KI, Lee MY, Getchell KM, So K, Hirshman MF \& Goodyear LJ 2014 Exercise before and during pregnancy prevents the deleterious effects of maternal high-fat feeding on metabolic health of male offspring. Diabetes 64 427-433. (doi:10.2337/db13-1848)

Sunny NE, Parks EJ, Browning JD \& Burgess SC 2011 Excessive hepatic mitochondrial TCA cycle and gluconeogenesis in humans with nonalcoholic fatty liver disease. Cell Metabolism 14 804-810.

Swift DL, Johannsen NM, Lavie CJ, Earnest CP \& Church TS 2014 The role of exercise and physical activity in weight loss and maintenance. Progress in Cardiovascular Diseases 56 441-447. (doi:10.1016/j.pcad. 2013.09.012)

Valckx SD, Arias-Alvarez M, De Pauw I, Fievez V, Vlaeminck B, Fransen E, Bols PE \& Leroy JL 2014a Fatty acid composition of the follicular fluid of normal weight, overweight and obese women undergoing assisted reproductive treatment: a descriptive cross-sectional study. Reproductive Biology and Endocrinology 12 13. (doi:10.1186/1477-7827-12-13)

Valckx SD, Van Hoeck V, Arias-Alvarez M, Maillo V, Lopez-Cardona AP, Gutierrez-Adan A, Berth M, Cortvrindt R, Bols PE \& Leroy JL 2014b Elevated non-esterified fatty acid concentrations during in vitro murine follicle growth alter follicular physiology and reduce oocyte developmental competence. Fertility and Sterility 102 1769-1776.e1. (doi:10. 1016/j.fertnstert.2014.08.018)

Valsangkar D \& Downs SM 2013 A requirement for fatty acid oxidation in the hormone-induced meiotic maturation of mouse oocytes. Biology of Reproduction 89 43. (doi:10.1095/biolreprod.113.109058)
Vega CC, Reyes-Castro LA, Bautista CJ, Larrea F, Nathanielsz PW \& Zambrano E 2013 Exercise in obese female rats has beneficial effects on maternal and male and female offspring metabolism. International Journal of Obesity 39 712-719. (doi:10.1038/ijo.2013.150)

Wagener A, Schmitt AO \& Brockmann GA 2012 Early and late onset of voluntary exercise have differential effects on the metabolic syndrome in an obese mouse model. Experimental and Clinical Endocrinology \& Diabetes 120 591-597. (doi:10.1055/s-0032-1321727)

Wang Q, Ratchford AM, Chi MM, Schoeller E, Frolova A, Schedl T \& Moley KH 2009 Maternal diabetes causes mitochondrial dysfunction and meiotic defects in murine oocytes. Molecular Endocrinology 23 1603-1612. (doi:10.1210/me.2009-0033)

Wang Q, Chi MM \& Moley KH 2012 Live imaging reveals the link between decreased glucose uptake in ovarian cumulus cells and impaired oocyte quality in female diabetic mice. Endocrinology 153 1984-1989. (doi:10.1210/en.2011-1815)

Wittemer C, OhI J, Bailly M, Bettahar-Lebugle K \& Nisand I 2000 Does body mass index of infertile women have an impact on IVF procedure and outcome? Journal of Assisted Reproduction and Genetics 17 547-552. (doi:10.1023/A:1026477628723)

Wu LL, Dunning KR, Yang X, Russell DL, Lane M, Norman RJ \& Robker RL 2010 High-fat diet causes lipotoxicity responses in cumulus-oocyte complexes and decreased fertilization rates. Endocrinology 151 5438-5445. (doi:10.1210/en.2010-0551)

Wu LL, Russell DL, Norman RJ \& Robker RL 2012 Endoplasmic reticulum (ER) stress in cumulus-oocyte complexes impairs pentraxin-3 secretion, mitochondrial membrane potential (DeltaPsi m), and embryo development. Molecular Endocrinology 26 562-573. (doi:10.1210/me.2011-1362)

Yan Z, Lira VA \& Greene NP 2012 Exercise training-induced regulation of mitochondrial quality. Exercise and Sport Sciences Reviews 40 159-164. (doi:10.1097/JES.0b013e3182575599)

Yang X, Wu LL, Chura LR, Liang X, Lane M, Norman RJ \& Robker RL 2012 Exposure to lipid-rich follicular fluid is associated with endoplasmic reticulum stress and impaired oocyte maturation in cumulus-oocyte complexes. Fertility and Sterility 97 1438-1443. (doi:10.1016/j.fertnstert.2012.02.034)

Received 7 September 2015

First decision 30 September 2015

Revised manuscript received 2 December 2015

Accepted 22 December 2015 